J. Clin. Chem. Clin. Biochem.

Vol. 14, 1976, pp. 537-542

\title{
Vergleichende Untersuchungen zur Erythropoetin(ESF)-Konzentrierung im Harn mit Ultrafiltration (Diaflo-Verfahren) und mit der Harndialyse gegen höherosmolare Substanzen (Carbowax)
}

\author{
Von E. Schulz und H. Cissée
}

Medizinische Universitäts-Poliklinik und Medizinische Klinịk (Direktor: Prof. Dr. med. W. Kaufmann) Köln-Merheim

(Eingegangen am 24. April/3. September 1976)

Zusammenfassung: Es wird das Diaflo-Ultrafiltrationsverfahren mit den Polyionenmembranen UM 10, PM 10 und PM 30 zur Erythropoetin-Konzentrierung im Harn mit der Harndialyse in Visking-Schläuchen gegen Carbowax verglichen. Die Wiederfindung von tierischem Erythropoetin im menschlichen Harn beträgt bei Ultrafiltration mit der UM 10-Membran durchschnittlich 84\%, mit der PM 10-Membran 62\%, mit der PM 30-Membran 17\% und bei Harndialyse $71 \%$. Die UM 10-Konzentrate werden von den Mäusen im Vergleich zu den Konzentraten nach Harndialyse wesentlich schlechter vertragen. Auch die Injektion der Konzentrate von Nierenpatienten führt zu einer höheren Absterberate der Tiere. Die Verträglichkeit der Konzentrate wird durch Verabfolgung gleicher Mengen Harnbestandteile in einem um das Dreifache vergrößerten Volumen bei Fraktionierung der Dosis auf drei Injektionen erheblich verbessert. Die polyglobulen Mäuse tolerieren Harnkonzentrate, wenn pro Maus nicht mehr als $6 \%$ eines Gesamtkonzentrats von einem 24-Stunden-Harn intraperitoneal injiziert werden. Bei stark toxischer Wirkung ist bei den Tieren mit einer zu niedrigen Eiseninkorporationsrate zu rechnen.

Comparative experiments on erythropoietin concentrations in ultrafiltered urine (Diaflo-process) and urine dialysis with higher osmolar substances (Carbowax)

Summary: The Diaflo-ultrafiltration process with polyion membranes, UM 10, PM 10, and PM 30 for the determination of erythropoietin concentration in urine is compared with the method of urine dialysis in Visking tubes against Carbowax. The average regain of animal erythropoietin in human urine by ultrafiltration with the UM 10membrane is $84 \%$, with the PM 10 -membrane $62 \%$, with the PM 30 -membrane $17 \%$ and with urine dialysis $71 \%$. Mice show a lower toleration for the UM 10 concentrates than for the concentrates prepared by urine dialysis. The concentrates of kidney patients also cause a higher mortality in the animals. The concentrates are tolerated when equal amounts of urine components are incorporated in a three times larger volume and this is divided into three separate injections. Mice with polyglobulia tolerate urine concentrates when not more than $6 \%$ of a whole concentrate of a 24-hours-urine per mouse is injected in the peritoneum. There is a low iron incorporation rate in the mice that show a strongly toxic reaction.

\section{Einleitung}

Das besondere Problem der Erythropoetin-Bestimmung im Harn von Normalpersonen und Nierenpatienten ist die geringe Erythropoetin-Konzentration, die sich nur nach Harneinengung nachweisen läßt. Dazu wurden verschiedene Methoden mit Pervaporation (1, 2), Gefriertrocknung $(3,4)$ und Dialyse gegen höherosmolare Substanžen eingesetzt $(3,5,6,7)$. Aḷ zur Zeit effektivstes Harneinengungsverfahren für Eiweißkörper erwies sich in den letzten Jahren nach Pollak (8) und Renner (9) die Ultrafiltration mit Polyionenmembranen (Diaflo: Verfahren), wobei Ichiki \& Lange (10) erstmals das
Hollow-Fiber-Dialysesystem der Firma AMICON für die Erythropoetinkonzentrierung im Harn untersuchten.

Wir führten Wiederfindungsversuche mit drei verschiedenen Polyelektrolyt-Membranen der Firma AMICON im Vergleich zur Harndialyse gegen höherosmolare Substanzen durch. Außerdem prüften wir die Verträglichkeit der verschiedenen Harnkonzentrate bei polyglobulen Mäusen sowie die Auswirkung von toxischen Reaktionen auf das Erythropoetin-Bestimmungsergebnis, d. h. auf die ${ }^{59} \mathrm{Fe}$-Incorporationsrate bei der polyglobulen Maus. 


\section{Wiederfindungsversuche}

\section{Methodisches Vorgehen}

Eine männliche gesunde Versuchsperson sammelte über 10 Tage den Urin in Polyäthylenflaschen, wobei jede Blasenentleerung sofort cingefroren wurde. Zur Verhinderung eincr baktcriellen Zerstörung von Erythropoetin befand sich in jeder Flasche fur eine 24-Stunden-Sammelperiode ein Antibioticagemisch aus $125 \mathrm{mg}$ Streptomycin und $275 \mathrm{mg}$ Penicillin G. Nach Auftauen wurde der gesamte Urin gepoolt und in 20 gleiche Portionen aufgeteilt. Jede Einzelportion entspricht somit einer 12 stündigen Sammelperiode. Vier Portionen froren wir ohne Erythropoetinzusatz crneut bei $-20^{\circ} \mathrm{C}$ ein. Bei vier weiteren Gruppen von je vier Flaschen wurden vorher pro Flasche 3, 6, 11 bzw. $30 \mathrm{E}$. Erythropoetin der Connaught-Laboratories (Charge 149/1) zugesetzt.

\section{Harneinengung mit dem Diaflo-Ultrafiltrationsverfahren} Die Harneinengung mit dem Diaflo-Verfahren der Firma AMICON $^{1}$ ) erfolgte mit den Membrantypen UM 10, PM 10 und PM 30 in der Ultrafiltrationszelle Modell 202. Die beiden erstgenannten Typen besitzen eine Ausschlußgrenze von einem Molekulargewicht von 10000 und die PM 30 von 30 000. Da das Molekulargewicht von Erythropoetin im Harn zwischen 20000 und 33000 angegeben wird (11-13): müßte Erythropoetin durch die UM 10- und PM 10 konzentriert werden.

Der Harn wurde erneut aufgetaut, mit Whatman-Papier filtriert und bei $+4{ }^{\circ} \mathrm{C}$ durch nachgereinigten Stickstoff aus einem Vorratsbehälter in der Ultrafiltrationszelle durch die Ultrafiltrationsmembran gepreßt. Nach Entfernen des Harnkonzentrats spülten wir die Membranoberfläche mit $5 \mathrm{ml}$ isotonischer Natriumchlorid-Lösung nach. Harnkonzentrat und Spülflüssigkeit ergaben ein Gesamtkonzentrat von $22 \mathrm{ml}$.

\section{Harndialyse gegen höherosmolare Substanzen}

Zur Harnkonzentrierung mit der Harndialyse gegen höherosmolare Substanzen benutzten wir Visking-Dialysierschläuche (Typ 1/7/8 SS) und Carbowax $20 \mathrm{M}$ (Union Carbide Corporation, Seattle, Washington). Der mittlere Porenradius dieser Schläuche beträgt 2,4 nm, so daß das Erythropoetin mit einem Molekülradius von $3,3 \mathrm{~nm}$ (13) nicht diffundieren dürfte. Um den mit Harn gefüllten Schlauch wurden Carbowax-Plättchen gepackt. Wir engten die 12-Stunden-Urine auf 3 bis $8 \mathrm{ml}$ ein und spülten die Schläuche mit 13 bis $19 \mathrm{ml}$ isotonischer NatriumchloridLösung durch wiederholtes Ausstreichen möglichst gründlich nach, wobei wir die Spülflüssigkeit dem Konzentrat hinzufügten, das somit ebenfalls auf $22 \mathrm{ml}$ aufgefüllt wurde. Sämtliche Konzentrate wurden mit $500 \mathrm{~g} 20$ Minuten lang zentrifugiert und der Überstand bei $-20^{\circ} \mathrm{C}$ bis zur Erythropoetin-Bestimmung eingefroren.

\section{Erythropoetin-Bestimmung}

Die Erythropoetin-Bestimmung erfolgte mit der Methode der posthypoxischen polyglobulen NMRI-Maus $(14,15)$. Am 6. und 7. Tag nach Beendigung der Hypoxie verabfolgten wir den Mäusen jeweils $1 \mathrm{ml}$ Harnkonzentrat intraperitoneal und am 8. Tag $1 \mu \mathrm{Ci}$ ${ }^{59} \mathrm{Fe}$ intravenös und bestimmten am 10 . Tag die 48 -Stunden${ }^{59} \mathrm{Fe}-$ Inkorporationsrate (16). Die von den $2 \mathrm{ml}$ pro Maus rechnerisch ermittelte vorgelegte Erythropoetinmenge $(0,27-2,7 \mathrm{E}$. Connaught-Erythropoetin) wurde in isotonischer $\mathrm{NaCl}-\mathrm{Lösung}$ gelöst Kontrolltieren nach dem gleichen Schema injiziert, so daß dic bei den Kontrollticren ermittelte ${ }^{59} \mathrm{Fe}$-Inkorporationsrate $=100 \%$ gesetzt werden konnte. Weitere Kontrolltiere bekamen anstelle des Harnkonzentrats $2 \mathrm{mil}$ isotonische $\mathrm{NaCl}$-Lösung intraperitoneal.

\footnotetext{
1 AMICON, Mechclaarstraat 11, Oosterhout (N. B.), Holland.
}

\section{Ergebnisse}

Spalte A der Tabelle 1 gibt die im 12-Stunden-Harn ohne Erythropoetin-Zusatz ermittelte ${ }^{59} \mathrm{Fe}$-Inkorporationsrate wieder. Nur die Werte nach Injektion des UM 10Konzentrats und des Visking/Carbowax-Konzentrats liegen signifikant über den $\mathrm{NaCl}-$ Kontrollen $(\mathrm{p}<0.001)$. Mit Hilfe einer Erythropoetin-Standard-B-Dosiswirkungskurve fanden wir im UM 10-Konzentrat 1,79 und im Visking/Carbonat-Konzentrat 1,76 E Erythropoetin/ 12 Stunden-Harn ( $=3,59$ bzw. 3,52 E./24-Stunden-Harn). Die Erythropoetin-Wiederfindung ist mit der UM 10Membran mit $84 \%$ am größten (Spalte F). Ein signifikanter Unterschied gegenüber dem 100\%-Wert besteht nur in Spalte E. Die Wiederfindung in den Visking/ Carbowax-Konzentraten beträgt durchschnittlich $71 \%$. Das PM 10-Konzentrat zeigt eine durchschnittliche Wiederfindung von 62\%. Der Unterschied ist in Spalte B und E gegenüber dem 100\%-Wert signifikant $(p<0,05)$. Die PM 30-Membran ist für Erythropoetin weitgehend permeabel. Die mittlere Einengungsdauer von $496 \mathrm{ml}$ Harn war bei den Visking/Carbowax-Konzentraten am längsten (Tab. 1, Spalte G).

\section{Vergleich der Verträglichkeit der Harnkonzentrate}

Über die Verträglichkeit der Harnkonzentrate bei Mäusen als limitierender Faktor für den Erythropoetin-Nachweis im Harn findet man in der Literatur keine Hinweise.

\section{Methodisches Vorgehen}

Bei 1148 polyglobulen Mäusen beurteilten wir die Verträglichkeit verschiedener Harnkonzentrate nach folgenden Gesichtspunkten:

\section{Kriterien der Toxizität}

1. o. B.: Glattes Fell, Bewegungsaktivität, Fressen und Trinken vergleichbar den $\mathrm{NaCl}$-Kontrollen.

2. Gering bis mäßig krank: Bewegungsaktivität gering bis mäßig-gradig eingeschränkt, etwas struppiges Fell, Tiere fressen und trinken noch weitgehend normal.

3. Sehr krank: Bewegungsaktivität weitgehend aufgehoben; struppiges Fell, Tiere schwitzen (Markierungen auf Fuchsinlösung zerfließen), fressen und trinken praktisch nicht mehr.

4. Exitus

Das Gewicht wurde am 10. posthypoxischen Tag festgestellt.

\section{Verwendete Harnkonzentrate und Injektionsschemata}

Die mit der UM 10-Membran bzw. mit Visking-Schläuchen gegen Carbowax eingeengten 12- und 24-Stunden-Urine stammen von verschiedenen Normalpersonen und Nierenpatienten. Zunächst spritzen wir von den auf $16 \mathrm{ml}$ eingeengten 12-Stunden-Urinen $2 \mathrm{ml}$, d. h. 12,5\% des Gesamtkonzentrats pro Maus intraperitoneal. Die Dosis wurde auf 2 Tage verteilt. Da wir beobachteten, daß die krank reagierenden Tiere mèist vorübergehend stark exsikkiert waren, verdünnten wir die Konzentrate in einer weiteren Versuchsserie mit isotonischer Natriumchlorid-Lösung auf $50 \mathrm{ml}$ und spritzten $6 \mathrm{ml}$ pro Maus, das sind $12 \%$ des Gesamtkonzentrates eines 12Stunden-Urins. Die $6 \mathrm{ml}$ wurdẹn über 3 Tage verteilt (15). 


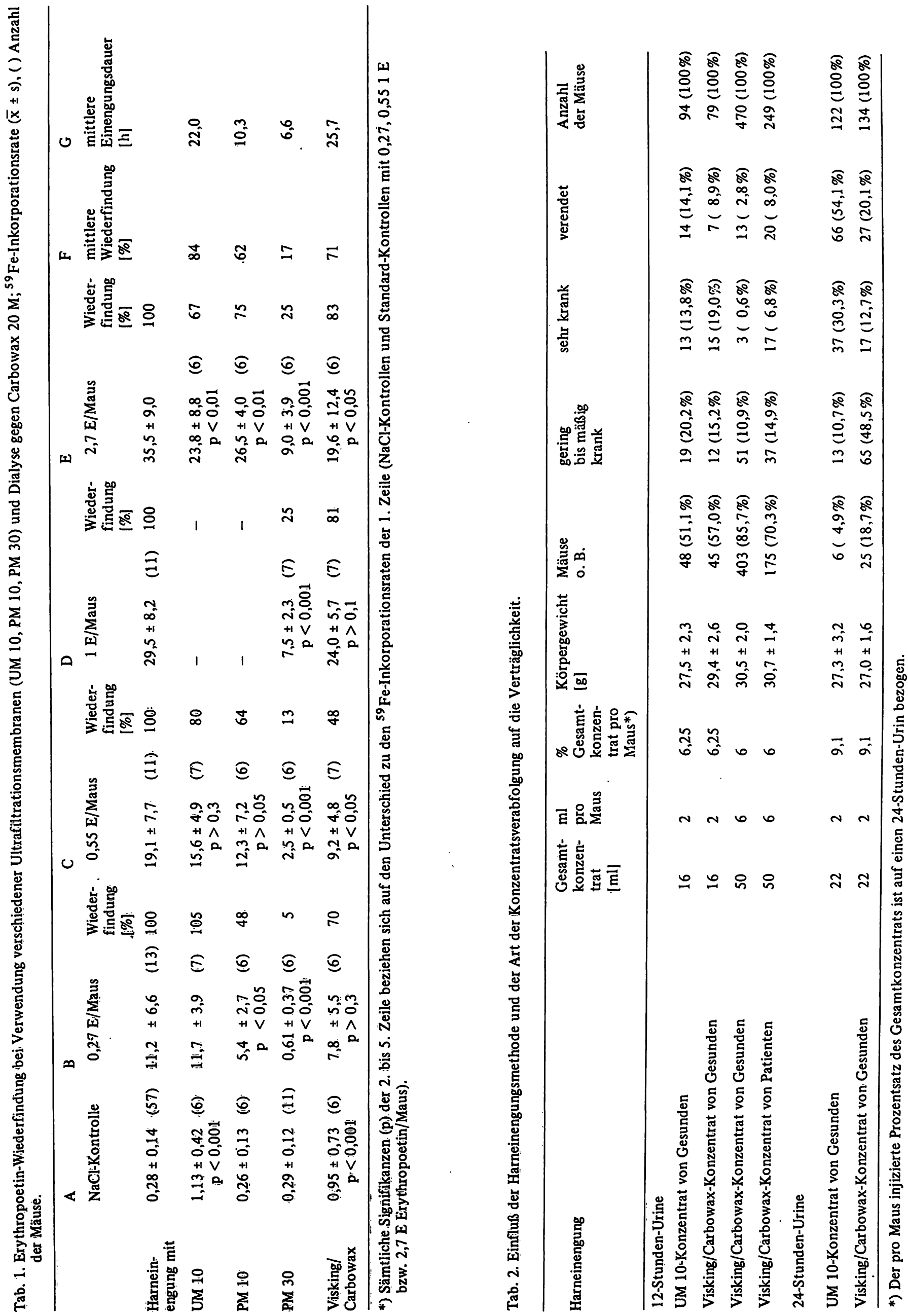


Von den 24-Stunden-Urin-Portionen gewannen wir ein Gesamtkonzentrat von $22 \mathrm{ml}$ und gaben den Mäusen $2 \mathrm{ml}(=9,1 \%$ des 24-Stunden-Gesamtkonzentrats). Hinsichtlich der pro Maus verabfolgten prozentualen Anteile vom Gesamtkonzentrat lassen sich dic 12-Stunden-Urinkonzentrate mit den 24-StundenUrinkonzentraten folgendermaßen vergleichen:

Diese Anteile betragen bei den 12-Stunden-Urinen 12,5 bzw. $12 \%$ des Gesamtkonzentrates von 16 bzw. $50 \mathrm{ml}$. Die pro Maus verabfolgten Harnbestandteile entsprechen somit 6,25 bzw. $6,0 \%$ eines gleichstark eingeengten 24-Stunden-Harns (Tab. 2, Spalte 3).

\section{Ergebnisse (Tab. 2)}

Die UM 10-Konzentrate werden von den Mäusen wesentlich schlechter vertragen als die Visking/Carbowax-Konzentrate (Zeile 1 und 2; 5 und 6). Die Absterberaté liegt nach UM 10-Konzentraten bei $54,1 \%$ und nach Visking/ Carbowax-Konzentraten bei 20,1\%. Die Verträglichkeit der Visking/Carbowax-Konzentrate läßt sich durch Verdünnen der pro Maus verabfolgten Harnbestandteile weiter bessern. Durch stärkeres Verdünnen der Konzentrate auf $50 \mathrm{ml}$ und Verabfolgung der Harnbestandteile in einem größeren Volumen über drei Tage senkten wir bei den 12-Stunden-Urinen die Mortalität von 8,9 auf $2,8 \%$ und die Zahl der sehr kranken Tiere von 19 auf $0,6 \%$ (Zeile 2 und 3). Die Visking/Carbowax-Konzentrate von Nierenpatienten werden schlechter als die Konzentrate von Gesunden toleriert (Zeile 3 und 4).

Neben einer besseren Verträglichkeit weist nach unseren Untersuchungen die fraktionierte Verabfolgung von Erythropoetin auf 3 anstelle 2 Einzeldosen höhere Eiseninkorporationsraten und somit eine größere Empfindlichkeit des biologischen Tests auf (16).

\section{Statistische Bearbeitung}

Mit dem $\chi^{2}$-Test wurden die in Tabelle 2 dargestellten Unterschiede auf Signifikanz geprüft. Dabei verglichen wir die Werte der Zeile 1 bis 3 miteinander, die Zeile 3 und 4, Zeile 5 und 6 sowie Zeile 2 und 6 . Sämtliche Unterschiede waren bei der Prüfung mit einer Irrtumswahrscheinlichkeit von $0,1 \%$ hoch signifikant.

\section{Körpergewicht}

Als Ausdruck der größeren Toxizität liegt das Körpergewicht der Mäuse nach Injektion der mit der UM 10Membran (Zeile 1) erhaltenen 12-Stunden-Harnkonzentrate und nach Injektion von beiden 24-Stunden=Harnkonzentraten (Zeile 5 und 6) mit 27 bis $27,5 \mathrm{~g}$ signifikant niedriger, verglichen mit den Mäusen nach Injektion von mit Visking/Carbowax erhaltenen Konzentraten der 12-Stunden-Urine (Zeile 2, 3 und 4; $p<0,001$ ).

\section{Toleranzgrenze der Maus gegenüber den verabfolgten Harnbestandteilen}

Den in Tabelle 2, Zeile 5 und 6, wiedergegebenen Mäusen wurden pro Maus 9,1\% eines 24-Stunden-Gesamtkonzentrats injiziert. Somit erhielten diese Tiere etwa ein Drittel mehr an Harnbestandteilen im Vergleich zu den Mäusen in Zeile 1 bis 3. Bei Hinzufugen von einem Drittel mehr an Harnbestandteilen steigt die Absterbequote der Tiere um mehr als das Doppelte an (vgl. Zeile 1 mit 5 und Zeile 2 mit Zeile 6). Die Toleranżgrenze ist somit erreicht, wenn mehr als $6 \%$ des Gesamtkonzentrats eines 24-StundenHarns pro Maus verabfolgt werden.

\section{Einfluß der Toxizität auf die ${ }^{59} \mathrm{Fe}$-Inkorporationsrate}

Die Angabe von Adamson et al. (3), daß Mäuse nach toxischen Urinkonzentraten eine verminderte ${ }^{59} \mathrm{Fe}$ Inkorporationsrate zeigen, können wir mit zwei eindrucksvollen Beobachtungen bestätigen (Tab. 3).

\section{Methodisches Vorgehen}

Der Urin einer 48-Stunden-Sammelperiode von zwei Patientinnen mit aplastischer Anämie wurde geteilt und eine Hälfte durch Ultrafiltration und die andere durch Dialyse gegen Carbowax auf $22 \mathrm{ml}$ eingeengt. Die Konzentrate spritzten wir 4 Gruppen von je 7 polyglobulen Mäusen, beobachteten đie Verträglichkeit und bestimmten die ${ }^{59} \mathrm{Fe}$-Inkorporationsrate.

\section{Ergebnisse}

Auf das UM 10-Konzentrat der Patientin Ch. erkrankten die Tiere: 5 starben, 2 überlebende waren sehr krank und wiesen eine ${ }^{59} \mathrm{Fe}$-Inkorporationsrate von nur $1 \%$ auf. Bei den mit dem Visking/Carbowax-Konzentrat der gleichen Patientin gespritzten Tieren bemerkten wir nur vorübergehend mäßige Krankheitssymptome und stellen eine ${ }^{59} \mathrm{Fe}$-Inkorporationsrate von $11,6 \%$ fest $(\mathrm{p}<0,001)$. Ein analoges Verhalten fanden wir mit Harnkonzentraten der Patientin K. $(p<0,005)$.

\section{Diskussion}

Die beste Wiederfindung von Erythropoetin-Zusätzen aus Schafplasma (Erythropoetin der Connaught-Laboratorien) im menschlichen Harn zeigte sich nach den Einengungsverfahren mit dem Diaflo-Ultrafiltrations-Gerät bei Anwendung der UM 10-Membran. Sie betrug im Mittel $84 \%$. Auch der Erythrypoetin-Nachweis im Harn ohne Zusätze war mit dieser Membran am höchsten. Die ErythropoetinExkretion pro 24 Stunden lag mit 3,95 ErythropoetinStandard-B-Einheiten in dem von Alexanian (6) und Adamson (3) bei gesunden Männern gefundenen Bereich (2,8 bis 4 E./24 Stunden). Als fast gleichwertig erwies sich das Visking-Carbowax-Einengungsverfahren. Adamson et al. fanden auch mit diesem Einengungsverfahren eine verlustlose Wiederfindung, wobei sie die relativ großen Membranoberflächen mindestens żweimal spülten 
und die Spülflüssigkeit erneut einengten (3). Von uns wurde dagegen die Membranoberfläche von ca. $900 \mathrm{~cm}^{2}$ nur einmal mit $20 \mathrm{ml}$ isotonischer Natriumchloridlösung gespült, was vielleicht die etwas geringere Ausbeute erklärt. Dem Vorteil der sehr kurzen Filtrationsdauer mit der PM 10-Membran steht die nicht so günstige Wiederfindung von $62 \%$ entgegen. Möglicherweise hat auch das axiale Verhältnis des Erythropoetin-Moleküls von 1:10 (17) für den Durchtritt durch eine Membran und damit für seine Wiedergewinnung hier eine Bedeutung. Bei Einengung mit der PM 30-Membran gehen $83 \%$ Erythropoetin verloren. .

Eine interessante Beobachtung machten Ichiki \& Lange (10), die einen Erythropoetin-reichen Harn eines stark anämischen Patienten mit paroxysmaler nächtlicher Hämoglobinurie mit dem Hollow-Fiber-System DC 2 der Firma AMICON konzentrierten und mit dem Erythropoetin-Gehalt des rohen Urins verglichen. Sie benutzten dabei die Dia-Fiber-Membran, Patrone H 1 DP 10, die nach Angabe des Herstellers im wesentlichen die Membraneigenschaften der von uns geprüften PM 10 besitzt. Bei zwei Versuchen war die Wiedergewinnung der Erythropoetin-Aktivität im konzentrierten Harn, berechnet auf das Ausgangsvolumen, höher als im rohen Urin (137 bzw. 102\%). Die Autoren erklärten die Wiedergewinnung über $100 \%$ damit, daß während der Harnkonzentrierung vermutlich ein niedermolekularer Erythropoetin-Inhibitor eliminiert wurde. In dem Hollow-FiberDialyseverfahren ist die Harnkonzentrierung durch Ultrafiltration variabel gekoppelt mit der Dialyse, z. B. gegen destilliertes Wasser, während wir nur die Ultrafiltration einsetzten. Ob diese Koppelung die Ursache für die höhere Erythropoetin-Ausbeute bei Ichiki \& Lange (10) ist, läßt sich nur durch vergleichende Untersuchungen beider Methoden entscheiden.

Die mit Ultrafiltrationsverfahren (Membrantyp UM 10) erhaltenen Konzentrate zeigen bei polyglobulen Mäusen gegenüber den in Visking-Schläuchen gegen
Carbowax gewonnenen Konzentraten eine schlechtere Verträglichkeit. Das zeigt sich besonders deutlich an der Absterberate nach Injektion von 24-StundenHarnkonzentraten. Durch die UM 10-Membranen werden möglicherweise toxische Harnfarbstoffe retiniert, da die UM 10-Konzentrate gegenüber allen übrigen Konzentraten eine dunkelbraune Farbe besitzen. Durch Verabfolgung der Harnbestandteile in einem größeren Flüssigkeitsvolumen ( $6 \mathrm{ml}$ anstelle $2 \mathrm{ml}$ pro Maus) läßt sich die Verträglichkeit der Visking/Carbowax-Konzentrate weiter verbessern und die Absterberate von 8,0 auf 2\% senken. Trotz des Verdünnens und der Fraktionierung der Gesamtdosis auf drei Einzeldosen werden die Visking/ Carbowax-Konzentrate von Nierenpatienten im Vergleich zu den Visking/Carbowax-Konzentraten von Gesunden von den Mäusen schlecht vertragen. Die Verabfolgung von $6 \%$ eines 24-Stunden-Gesamtkonzentrats pro Maus stellt bei unserer Mäuserasse die Toleranzgrenze für Harnkonzentrate dar.

Das Beachten toxischer Symptome bei den Mäusen ist für die Interpretation der Ergebnisse bedeutsam. Da nach unseren Untersuchungen die Wiederfindung in den UM 10-Konzentraten nicht schlechter ist als in den Visking/Carbowax-Konzentraten, müssen wir die um das 10 fache niedrigere Eiseninkorporationsrate bei zwei Versuchen (Tab. 3) auf die Toxizität der UM10-Konzentrate infolge Überschreitung der Toleranzgrenze bei den Mäusen zurückführen. Die Ergebnisse bei mit starken toxischen Symptomen reagierenden Tieren sind somit nicht oder nur mit größter Vorsicht zu bewerten.

\section{Danksagung}

Mit Unterstützung der Deutschen Forschungsgemeinschaft. Für die technische Assistenz danken wir Frau $R$. Roesch und Frl. E. Steiner.

Tab. 3. Verträglichkeit und ${ }^{59}$ Fe-Inkörporationsrațe $(\overline{\mathbf{x}} \pm \mathrm{s})$ bei Verabfolgung verschiedener Erythropoetin-haltiger Harnkonzentrate.

\begin{tabular}{|c|c|c|c|c|c|c|c|c|}
\hline & $\begin{array}{l}48-S t d . / 2= \\
\text { Urin- } \\
\text { volumen }\end{array}$ & $\begin{array}{l}\text { Gesamt- } \\
\text { konżẹn- } \\
\text { trat } \\
\text { [mi] }\end{array}$ & $\begin{array}{l}\text { ml } \\
\text { Konzen- } \\
\text { trat } \\
\text { pro } \\
\text { Maus }\end{array}$ & $\begin{array}{l}\text { Anzahl } \\
\text { d. Mäuse } \\
\text { zu Ver- } \\
\text { suchs- } \\
\text { Beginn }\end{array}$ & $\begin{array}{l}\text { gering } \\
\text { bis } \\
\text { mäßig } \\
\text { krank }\end{array}$ & $\begin{array}{l}\text { sehr } \\
\text { krank }\end{array}$ & verendet & $\begin{array}{l}{ }^{59} \mathrm{Fe}-\text { Inkorpora- } \\
\text { tionsrate }\end{array}$ \\
\hline UM 10-Konzentrat Ch. & 912 & 22 & 2 & 7 & - & 2 & 5 & $1,0 \pm 0,6$ \\
\hline Visking/Carbowax-K̇onzẹtrat Ch. & 912 & 22 & 2 & 7 & 7 & - & - & $11,6 \pm 5,4$ \\
\hline UM 10-Konzentrat $\mathrm{K}$. & 645 & 22 & 2 & 7 & - & 6 & 1 & $0,7 \pm 0,4$ \\
\hline Viskinig/Carbo wạax 10-Konzentrat K. & $64 \overline{5}$ & 22 & 2 & 7 & 7 & - & - & $6,7 \pm 4,2$ \\
\hline
\end{tabular}

\section{Literatur}

1. Lange, R. D., McCarthy, J. M. \& Gallagher, N. J. (1961), Arch. Intern. Med. 108, 850-858.

2. Lewis, J. P;; Gallagher, N. I., Carmody, S. E. \& Lange, R. D. (1964), Proc. Soc. Exper. Biol. Med. 116, 742-745.
3. Adamson, J. W., Alexanian, R., Martinez, C. \& Finch, C. A. (1966), Blood 28, 354-364.

4. Finne, P. H. (1965), Brit. Med. J., 697-699.

5. Adamson, J. W. (1968), Blood 32, 597-609. 
6. Alexanian, R. (1966), Blood 28, 344-353.

7. Alexanian, R., Vaughn, W. K. \& Ruchelman, M. W. (1967), J. Lab. Clin. Med. 70, 777-785.

8. Pollak, V. E., Gaizutis, M. \& Rezaian, J. (1968), J. Lab. Clin. Med. 71, 338-344.

9. Renner, E. (1969), Habilitationsschrift Köln.

10. Ichiki, A. T. \& Lange, R. D. (1974), Biochem. Med. 10, $50-60$.

11. Giovannini, E. \& Bocchini, V. (1975), Acta Haemat. 53, $75-81$.
12. Lewis, J. P., Nẹal, W. A., Welch, E. T., Lewis, W. C., Dubose, C. M., Wright, C. S. \& Smith, L. L. (1973), Proc. Soc. Exper. Biol. Med. 142, 293-298.

13. O'Sullivan, M. B., Chiba, Y., Glēich, G. J. \& Linman, J. W. (1970), J. Lab. Clin. Med. 75, 771-779.

14. Cotes, M. \& Bangham, D. R. (1961), Nature 191, 1065-1067.

15. Schulz, E. \& Cissée, H. (1976), diese Z. 14, 557-559.

16. Schulz, E. (1972), Habilitationsschrift Köln.

17. Rosse, W. F., Berry, R. J. \& Waldman, T. A. (1963), J. Clin. Invest. $42,124-129$.

Priv.-Doz. Dr. med. Ekkehard Schulz Vinzenz-Pallotti-Hospital İnnere Abteilung

Vinzenz-Pallotti-Hospital

D-5060 Bergisch Gladbach 1 